Raf. J. Sci., Vol. 24, No.1 pp. 159-164, 2013

\title{
Calculating the Acceleration of the Universeby Using the Constant Time Hyper Surfaces
}

\author{
Muayyad A. Al-Obayde \\ Department of Physics \\ College of Science \\ University of Mosul \\ E-mail: malobayde@yahoo.com
}

(Received 7/3/2012;Accepted 27/5/2012)

\begin{abstract}
In this work, as the growth of the universe was treated as a discrete step evolution of a black hole. The speed of the evolution of the universe was found to be increased exponentially with the number of the step of the evolution. This result shows a good agreement with the observational evidence of the accelerated universe.
\end{abstract}

Keywords: accelerated universe, general relativity, constant time hypersurface.

\section{مسلب تهجل الكهن بلستخدل اللملوح الفولة ذات الزمن الثلبت}

\section{المالخص}

عولج تنلهي الكون بوصفه تنلميا متدرجا مفصلا لتب لسود. ووجد انسرعة النمو تزداد لسيا مع رق م تدرج النمو. ؤظظهرت النتيجة توافقا جيدا مع الدلال الارصادية للكون المعل. الكاملت الدالة: الكون المعطل، النبية العلمة، للنطوح الففرطة ذات الزمن الثابت.

The surprised discovery of cosmic acceleration (Riess et al., 1998) (Perlmutter et al., 1999), gives rise to a big challenge to theorists and cosmologists. So, many suggestions have been given to explain it. Most of these suggestions are based on the idea of the existence of repulsive gravity that could arise from some kind of vacuum energy with a negative gravitational pressure. Such energy is dark, and, may be, related to the cosmological constant, which added by Einstein to the field equations to stop the time evolution of the universe. The arguments for and recent progress made towards understanding the nature of dark energy was discussed in details (Copeland et al., 2006). Another suggestions are that the acceleration of the universe is due to a new long-range forces, the smallness of neutrino 
masses, it may signal that general relativity breaks down on cosmological scales and must be replaced (Frieman et al., 2008 ), and the acceleration is caused by quintessence in the form of a scalar field slowly evolving down a potential (Linder, 2007). An alternative approaches to the problem of accelerated universe use the particle creation process due to the expenses of the gravitational field or the creation of dark matter particle at the expenses of $\Lambda$-decay (de Campos, 2007)

This work suggests an explanation based, firstly, on the assumption that the universe is a black hole, and secondly, on previous results (Alobayde,1995) obtained from treating the particle creation by strong gravitational field, as a transformation of virtual particle described with $(\Delta E \Delta t<\hbar)$ to real particles described with $(\Delta E \Delta t \geq \hbar)$.

\section{Particle creation and the time evolution of the universe}

To investigate the question: Is the universe itself a black hole ?, (Pathria, 1972) assumed, in addition of the internal observer, an outside observer to get two views of the universe. For the inside observer the geometry of the space-time is governed by the Robertson-Walker metric, and, because the expansion of the universe is isotropic, the space-time, as observed from outside the universe, is governed by a static Schwarzschild metric. From equating the scalar curvature $\mathrm{R}(\mathrm{t})$ of the interior observer with Schwarzschild radius $\mathrm{R}_{\mathrm{s}}$ of the exterior observer at condition of closed universe $\mathrm{k}=+1$, and the presence of the cosmological constant in Einstein field equation, Pathria found that the universe is indeed a black hole. Accordingly, we can suggest that the present universe started its evolution from singularity surrounded by vacuum and stays like that. In quantum field theory in curved space-time, the vacuum state in flat space-time, is defined with the annihilation operator $(\alpha)$ as $(\alpha / 0>=0)$, and the creation operation is a direct result of the variation of the definition of the annihilation operator in different spaces. Consequently, the vacuum of the flat space time, should not be a vacuum in curved space-time, which means that the particle creation is caused by the gravitational field, or by the curvature of the space-time. So, in his pioneer work (Hawking, 1975) Hawking showed that quantum mechanical effects cause black holes to create and emit particles, as if they are hot bodies, and the creation rate depends on the mass of the black hole.

Pathria's and Hawking's results make it possible to treat the universe evolution, from the first singularity at $t_{0}$ which has the gravitational radius $\left(\mathrm{R}_{0}\right)$, as a growth of a black hole surrounded by a quantum vacuum .

In previous work (Alobayde, 1995) used the Schwarzschild metric to describe the spherical symmetric gravitational field, the dependence of the gravitational red shift on the time component of the metric, and the assumption that the vacuum is fill with virtual particles defined as $(\Delta E \Delta t<\hbar)$ to prove that the strong gravitational field whose gravitational red shift $(\mathrm{z} \geq 1)$ transform the virtual particles to real particles defined as $(\Delta E \Delta t>\hbar)$ at

$$
R \leq \frac{4 R_{s}}{3}
$$


Then using this result to suggest a description of the growth of a non-rotating uncharged black hole (Altaie and Aziz, 1998), and to derive a relation for calculating the velocity of expansion of the universe (Aziz, 1999) as fllows:

The gravitational radius of the universe at its beginning at $\left(\mathrm{t}_{0}\right)$ is $\left(\mathrm{R}_{0}\right)$ defined with

$$
R_{0}=\frac{2 G M_{0}}{c^{2}}
$$

$\mathrm{M}_{0}$ is the initial energy -mass of the universe.

The created particles increase $\left(\mathrm{M}_{0}\right)$ to $\left(\mathrm{M}_{1}\right)$, and so increases $\left(\mathrm{R}_{0}\right)$ to $\left(\mathrm{R}_{1}\right)$ such that

$$
R_{1}=\frac{4}{3} R_{0}
$$

In $\mathrm{Eq}(3)$ we just use the equality of (1)

From (3) and (2) we get

$$
M_{1}=\frac{4}{3} M_{0}
$$

For $\mathrm{N}$ - successive creation operations we can get the general relations

$$
\begin{aligned}
R_{n} & =\left(\frac{4}{3}\right)^{n} R_{0} \\
M_{n} & =\left(\frac{4}{3}\right)^{n} M_{0}
\end{aligned}
$$

As in the previous works, in this suggestion, we suppose that the created particles by the strong gravitational field of the initial state of the universe, which is black hole, added to the black hole and causes an increase in its Schwarzschild radius $\left(R_{s}\right)$. The variation of the radius $\left(\Delta \mathrm{R}_{\mathrm{s}}\right)$ is proportional to the added mass $(\Delta \mathrm{M})$. Then another particle creation takes place with the new gravitational radius and the created mass is added to the black hole and causes an increase in $\left(R_{s}\right)$, and so on. The time interval $\left(\Delta_{n} t\right)$ between any two successive radius $\left(R_{n}, R_{n+1}\right)$ is governed by the energy-time uncertainty. The event horizons, which correspond to each successive Schwarzschild radius considered as a constant time hyper surfaces (CTH's) labeled by its own time $\left(t_{n}, t_{n+1}\right)$. Consequently, the time evolution of the universe takes place as a successive of such process. This means that the universe expansion is a successive particle creation processes appears as a successive (CTH's) as shown in Fig (1). So, the expansion of the universe is a discrete time evolution. 


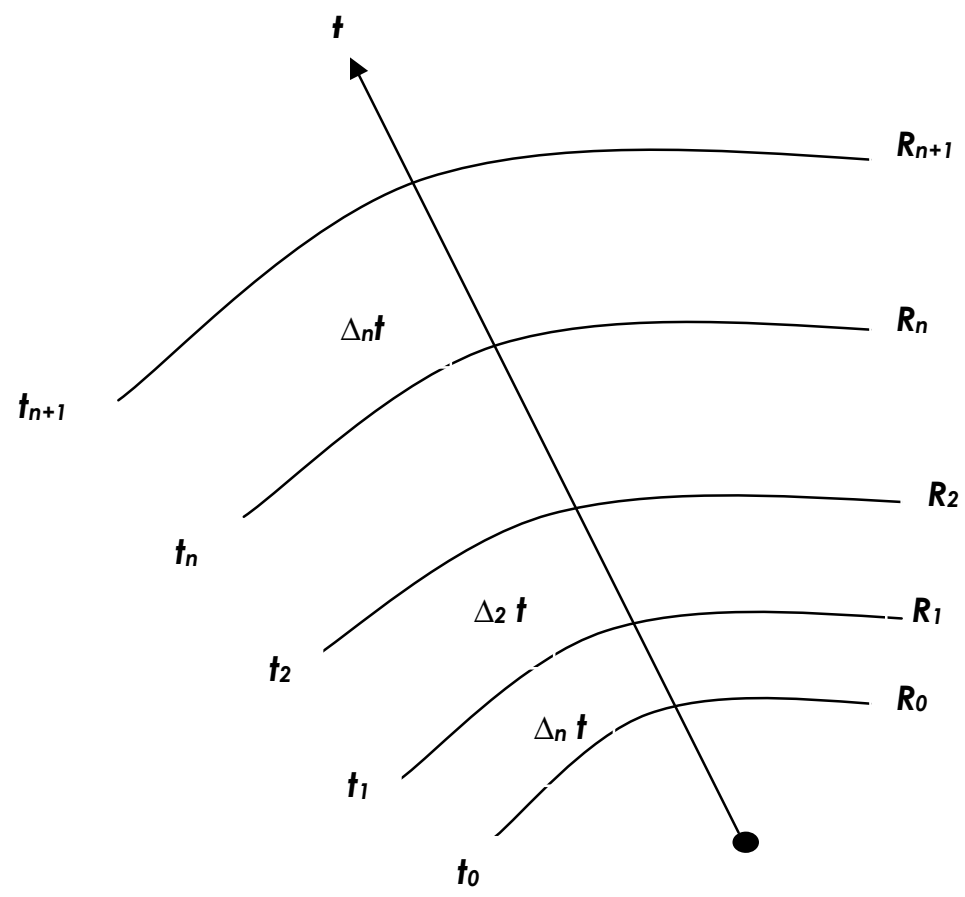

Fig. 1: successive constant time hypersurfaces

It is clear from equations (5) and (6) that the successive creation operations do not change the components $\left(\mathrm{g}_{00}\right)$ and $\left(\mathrm{g}_{11}\right)$ of Schwarzschild metric, which mean that the relation between the proper and the local time doesn't change in the successive creation operations. The rate of expansion of the universe can be calculated at any time interval from the ratio of the change in $\left(\mathrm{R}_{\mathrm{s}}\right)$, of two successive (CTH's), to the time interval between them $\left(\frac{R_{n+1}-R_{n}}{t_{n+1}-t_{n}}\right)$.

The time duration $(\Delta \mathrm{t})$ of any physical quantum process involving a change in the energy $(\Delta \mathrm{E})$ governed by Heisenberg principle of uncertainty. So the time duration $(\Delta \mathrm{t})$ between two successive $\mathrm{CTH}$, is

$$
\Delta t=\frac{\hbar}{\Delta M c^{2}}
$$

Where $(\Delta \mathrm{M})$ is the mass difference between two successive states.

Let us take two successive black hole states $(n, n+1)$, and consider $\Delta_{n} t$ as the time duration from state $(n)$ to the state $(n+1)$. Then, according to (5), the growth from $\left(R_{n}\right.$ to $\left.R_{n+1}\right)$ gives the variation $\left(\Delta_{n} R\right)$ as

$$
\Delta_{n} R=R_{n+1}-R_{n}=\left(\frac{4}{3}\right)^{n}\left(\frac{R_{0}}{3}\right)
$$

And from (7) this variation takes the time

$$
\Delta_{n} t=\frac{\hbar}{\left(M_{n+1}-M_{n}\right) c^{2}}=\left(\frac{4}{3}\right)^{-n}\left(\frac{M_{0}}{3}\right)^{-1}\left(\frac{\hbar}{c^{2}}\right)
$$


The equations (8) and (9) show that the value of the increase in the Schwarzschild radius, for successive states, is not constant but proportional to the serial number (n). And the time interval between successive CTHs varies inversely with (n). So, the speed of transition from any Schwarzschild radius $R_{n}$ to the next one $R_{n+1}$ during the time $\left(\Delta_{n} t\right)$ is

$$
v_{n}=\frac{\Delta_{n} R}{\Delta_{n} t}=\left(\frac{4}{3}\right)^{2 n}\left(\frac{M_{0} R_{0}}{9}\right)\left(\frac{\hbar}{c^{2}}\right)^{-1}
$$

And by means of the initial energy is

$$
v_{n}=\left(\frac{4}{3}\right)^{2 n}\left(\frac{E_{0} R_{0}}{9 \hbar}\right)
$$

From equations (2) and (10) we can write

$$
v_{n}=\left(\frac{4}{3}\right)^{2 n}\left(\frac{R_{0}^{2}}{18}\right)\left(\frac{c^{4}}{\hbar G}\right)
$$

Or

$$
v_{n}=\left(\frac{4}{3}\right)^{2 n}\left(\frac{2 M_{0}^{2}}{9}\right)\left(\frac{G}{\hbar}\right)
$$

It is clear from equations (11) and (12) that the speed of transition of the Schwarzschild radius is proportional to power $(2 n)$, and its value depends on the assumed initial mass and its corresponding Schwarzschild radius.

\section{RESULTS AND DISCUSSION}

The spatial evolution of the universe is discrete, appears as steps, with different spatial separations. As equations (8) and (9) show, these steps are separated by time intervals which are discrete and not unique for different steps. So every transition has its own speed, which is increasing as in equation (10). The energy-mass of the universe is increasing as in equation (6). Consequently, the universe started as a small black hole and grow as a black hole. The event horizon of every black hole is a CTH.

In agreement with the recent observational result of the expanding universe, comparing between the speeds of expansions at different time intervals, one can see that the expansion of the universe is accelerating.

As in the case of the energy difference between quantum energy levels in its relation with the quantum number (n), the time intervals decrease with the serial number (n), and the speed of expansion increases rapidly with the power (2n). So, the discrete growth of the universe should appear as it continues for large (n).

It is clear from the above results that the rate of expansion of the universe depends on its initial state, mass and radius. So, according to (Hawking, 1975), the initial mass of the universe should not be very small, because it should evaporate before starting its growth. The variation of the time intervals between successive steps of evolution certify that the Hubble's constant is time dependent. 
Knowing the initial and the present mass or radius of the universe can give the number (n) and so the age of the universe.

In spite of the agreement of eq. (10) with the recent observation of the accelerated universe, the suggested evolution faced difficult questions about the very fast expansion, and the observed structure of the universe in the interior of the black hole. The possible answers are that we live inside a universe enclosed with a very strong gravitational spherical shell seems like black hole to the exterior observer, and it is a close Robertson-Walker expanding universe for the interior observer. Consequently, if we take in consideration that both, the interior and exterior observers, are in approximately flat space-time then the very fast expansion resulted from the high gravitational red shift of the signals, that come from regions near the shell, is observed by the inside and outside observers.

\section{REFERENCES}

Alobayde, M.A. (1995). On Particle creation by strong gravitational fields. M.Sc. Thesis University of Mosul, Dept. of Physics - Iraq.

Altaie, M. B., Aziz, M. (1998). CTHs structure for non rotating black hole. J. Edu. Sci., 29, 9298.

Aziz, M. (1999). Relation for calculating the speed of the universe expansion according to the CTHs model. J. Edu. Sci., 39, 36-40.

Copeland, E.J.; Sami, M.; Tsujikawa, S. (2006). Dynamics of dark energy. Int. J. Mod. Phys. D15, 1753-1936.

de Campos, M. , (2007). Growing of the inhomogeneities and acceleration phase in universe with $\Lambda$ decay. Brazil. J. Phys., 37 (4), 1235-1237.

Frieman, J.A.; Turner, M.S.; Huterer, D. (2008). Dark energy and the accelerating universe. Annu. Rev. Astron. Astrophys., 46, 384-432.

Hawking, S. (1975). Particle creation by black holes. Commun. Math. Pys., 43, 199-220.

Linder, E.V. (2007). Theory challenges of the accelerating universe. J. Phys. A: Math.Theor., 40, 6697-6705.

Pathria, R.K. (1972). The universe as a black hole. Nature, 240, (5379), 298-299.

Perlmutter, S.; Aldering, G.; Goldhaber, G.; Knop, R.A.; Nugent, P.; Castro, P. G.; Destua, S. ; Fabbro, S.; Goobar, A. ; Groom, D. E.; Hook, M. ; Kim, A. G.; Kim, M. Y.; Lee, J. C., Nunes, N. J., Pain, R., Pennypacker, C. R., Quimby, R., Lidma, C., Ellis, R. S.; Irwin, M.; Mcmahon, R. G.; Ruiz-Lapuente, P.; Walton, N. ; Schaefer, B.B.; Boyle, J.; Filippenko, A. V.; Matheson, T.; Fruchter, A. S.; Panagia, N. ; Newberg, H. J. M. ; Ouch W. J. (1999). (The supernova cosmology project), The measurement of Omega and Lambda from 42 high redshift supernova. Astrophysical J., 517, 565-586.

Riess, A. G.; Filippenko, A. V.; Challis, P.; Clocchiatti, A.; Diercks, A. ; Garnavich, P. M.; Gilliland, R. L.; Hogan, C.J.; Jha, S.; Kirshner, R. P.; Leibundgut, B.; Phillips, M. M.; Reiss, D.; Schmidt, B. P.; Schommer, R. A.; R. Smith C.; Spyromili, J. ; Stubbs, C.; Suntzeff, N. B.; Tonry, J. ; (1998). Observational evidence from supernova for an accelerating universe and a cosmological constant. Astron. J., 116, 1009-1038. 